\title{
Perfil y número de médicos generales que requiere el país
}

\author{
O scar Román $\mathrm{A}^{1}$, Sabina Pineda $\mathrm{R}^{2 \mathrm{a}}$, Miriam Señoret $\mathrm{S}^{2 \mathrm{a}}$. \\ The profile and number of primary \\ care physicians required in Chile
}

Medical schools curricular planning aim to obtain a physician trained to work as general practitioner and the Chilean health reform, considers ambulatory primary care as the main axis of health care. However there is still a low interest among physicians to work in primary health care, where there are problems related to a low level of clinical resolution, clinical and administrative management deficiencies and a low level of leadership in health promotion. The causes of these deficiencies stem from university training, government policies and the great attraction that exerts the technological and specialized model of secondary and tertiary health care. We analyze the ideal profile that the general practitioner should have in our health care system and the possible solutions to primary health care problems. We also emphasize the need to coordinate the professional resource needs with university training, to reduce the existing gaps between medical training and professional practice (Rev Méd Chile 2007; 135: 1209-15).

(Key words: Curriculum; Health resources; Primary health care)

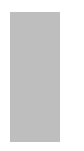
\footnotetext{
Desarrollo de las Personas.

aMatrona
}

Recibido el 25 de septiembre, 2006. Aceptado el 17 de enero, 2007.

${ }^{1}$ Facultad de Medicina, Universidad de Chile. ${ }^{2}$ Ministerio de Salud, División de Gestión y

L a proporción entre médicos generales (MG) y especialistas se ha modificado drásticamente en nuestro país en la última década, pues el predomino de $\mathrm{MG}$, estimado en $70 \%$ en los años 50, se ha invertido a favor de los especialistas. Según el Colegio Médico, en $1996^{1}$, 43\% de los médicos se autodefinían como MG, cifra que en el año 2004 alcanza sólo a 35\% de los médicos certificados en el sector público ${ }^{2}$. Si se restan los MG de las Municipalidades, la cifra cae a 15,8\% en el Sistema de Servicios de Salud ${ }^{2}$ (Tabla 1). Surgen de inmediato varias interrogantes:

Correspondencia a: Dr. Oscar Román A. Huelén 154 Depto 2. Santiago. Chile. E mail: oscarromanalemany@hotmail.com
1. ¿Por qué se ha producido este fenómeno?

2. ¿Cuáles serán las consecuencias para la atención de salud nacional, de acuerdo a las políticas implementadas por la reciente Refor$\mathrm{ma}^{3}$ que privilegian a la atención primaria de salud (APS) como eje del sistema?

3. ¿Cuáles podrían ser las posibles soluciones?

Trataremos de analizarlas en el mismo orden.

A. Causas de la menor proporción de médicos generales. La disminución de la proporción de MG tiene diversas raíces, que no han sido investigadas estadísticamente pero que, según encuestas de opinión y otros estudios focales, senian las siguientes:

a) Ausencia de carrera funcionaria. Los médicos, una vez ingresados a la APS, se rigen por el Estatuto de Salud Municipal ${ }^{4}$ y, por tanto, sólo 
Tabla 1. M édicos generales y especialistas en el país (2004)

\begin{tabular}{|c|c|c|}
\hline Total Sistema Público & $\mathrm{n}$ & $\%$ \\
\hline Total Médicos contratados & 10.906 & 100 \\
\hline Médicos Generales & 3.833 & 35,1 \\
\hline Médicos especialistas & 6.543 & 60,0 \\
\hline No determinados & 530 & 4,9 \\
\hline \multicolumn{3}{|l|}{ Total Servicios de Salud } \\
\hline Total Médicos de los SS & 8.610 & 100 \\
\hline Médicos generales & 1.364 & 15,8 \\
\hline Médicos especialistas (con o sin certif) & 6.543 & 76,0 \\
\hline Médicos en comisión estudios & 531 & 6,2 \\
\hline No determinados & 172 & 2,0 \\
\hline \multicolumn{3}{|l|}{ Médicos en APS Municipalidades } \\
\hline Población a atender & Médicos 44Hrs & Rel. $44 \mathrm{hrs} / 10.000$ beneficiarios \\
\hline 9,759.327 & 1.844 & 1,84 \\
\hline Requerimiento ideal & 3.140 & 3,14 \\
\hline Déficit & 1.296 & \\
\hline
\end{tabular}

pueden acceder a los escasos cargos directivos superiores, previo acuerdo de los Consejos Municipales o de las Corporaciones de Salud que dependen de ellos. Sin embargo, pueden hacer carrera hacia la especialización, concursando a la primera etapa o Etapa de Formación y Destinación (EFD) del nuevo Estatuto Médico Funcionario establecido por la Ley $19.664^{5}$. De acuerdo a él, después de 3 años de función asistencial en la APS, los MG pueden optar a becas de especialización del Ministerio de Salud, que se cumplen en las Universidades que hayan suscrito convenios con los Servicios de Salud de los que dependen las Municipalidades contratantes. Estas becas duran 1 a 3 años, al cabo de los cuales, el médico, ya especializado, debe ejercer en los establecimientos del Servicio de origen durante el doble de años que duró la beca, o sea un lapso de 4 a 6 años. En consecuencia, estas becas no apuntan a una verdadera carrera funcionaria en la APS sino sólo a la especialización, y por tanto, al abandono de la atención ambulatoria primaria.

b) Escasez de cupos en las plantas de las Municipalidades y Sistema de Salud. En las municipalidades, los cupos están bastante cubiertos y se podría suponer que el número de $\mathrm{MG}$ es adecuado a las necesidades. Sin embargo, ello no es así pues si nos atenemos a las recomendaciones internacionales, que establecen para la APS un número de 3,14 jornadas médicas completas (44 horas) por cada 10.000 beneficiarios (en nuestro caso pertenecientes a FONASA), resulta que para atender a una población nacional al 2006 de 9.759.327 beneficiarios se necesitarían 3.140 médicos y sólo están contratados 2.363 y de ellos, sólo 1.844 con jornada completa. En consecuencia, existe un déficit de 1.296 médicos de jornada completa (Tabla 1). Esta brecha se ha ido llenando, en parte, por médicos inmigrantes.

En el Sistema de Servicios de Salud, el panorama es similar, por cuanto de los poco más de 660 egresados anuales, concursan entre $250 \mathrm{y}$ 300 a los cupos del Ciclo de Destinación, ahora EFD. De ellos, sólo 120 a 150 son contratados anualmente por el Ministerio de Salud (Minsal), debido a que se les ofrecen sólo las vacantes dejadas por los MG de Zona que retornan a especializarse (Tabla 2).

c) Desinterés de los médicos egresados. Esta falta de interés es claramente perceptible, a pesar de las mejoras salariales ofrecidas por la APS municipal. Los médicos no han descubierto la 
Tabla 2. M édicos recién egresados que ingresan a ED F o a universidades. (C oncursos M insal)

\begin{tabular}{|lccc|}
\hline Año & Ingreso MG (EDF) & Ingreso a universidad & Retorno MG \\
\hline 2001 & 147 & 52 & 140 \\
2002 & 193 & 30 & 150 \\
2003 & 158 & - & 139 \\
2004 & 116 & 15 & 110 \\
2005 & 118 & 29 & 113 \\
2006 & 126 & 44 & 119 \\
Promedio & 143 & 34 & 108 \\
\hline
\end{tabular}

EDF: Etapa de destinación y formación (ley 19.664).

riqueza en aspectos antropológicos que se manifiestan en la APS y valoran más los aspectos prácticos como la ausencia de un definido sistema de perfeccionamiento profesional, la escasez de tecnologías de apoyo diagnóstico-terapéutico, la carencia de apoyo de médicos experimentados y la falta de incentivos ${ }^{1,6,7}$.

d) Influencia de la educación médica universitaria. La mayor parte de la carrera de pretítulo transcurre en el ámbito hospitalario y no en el ambulatorio. Además, tiene un marcado acento en la especialización, sea porque la mayoría de los docentes son especialistas, sea porque los estudiantes se familiarizan con tecnologías complejas y pasan a depender de ellas pues brindan apoyo diagnóstico o terapéutico definidos ${ }^{8,9}$.

e) Carencia de un perfil adecuado a las necesidades de la APS. Si bien las Universidades han implementado hace 8 años una reforma importante de sus currículos de pregrado, tendiente a preparar un MG que responda a las necesidades del modelo bio-psico-social o premonitorio de riesgo, los actuales egresados aún acusan deficiencias en cuanto a una atención óptima en la APS de acuerdo a las necesidades de la Reforma en marcha ${ }^{10,11}$.

B. Efecto sobre el rol e importancia de la APS en la actual Reforma de salud. La política actual de salud establece que la APS es el centro de acción de la red de salud ${ }^{3}$. Ha dejado de ser tan sólo la primera instancia de atención o puerta de entrada al sistema, para constituirse básicamente en el centro ordenador de la red, ejecutando no sólo las atenciones curativas clásicas sino también, y prio- ritariamente, las de promoción, prevención y rehabilitación. Para ello debe contar con MG preparados integralmente para ejercer, coordinar y liderar las prestaciones de salud según ciclo de vida (niño, adulto, senescente). Además, la APS requiere un equipo multiprofesional completo y la participación de los especialistas para orientar y programar las acciones preventivas y de promoción de la salud. Por tanto, para lograr que la APS pueda constituirse en la base de acción sanitaria de la actual reforma, necesita un MG capaz de resolver los problemas clínicos de la enfermedad, establecer acciones de promoción y prevención del riesgo de enfermar, liderar al equipo de salud, gestionar una acción coordinada con los especialistas de la red y ejecutar las acciones administrativas y financieras necesarias ${ }^{3}$.

Se comprende que para realizar tales acciones se hace indispensable exigir un perfil integral al MG, quien debe ser formado por las Universidades de acuerdo a las necesidades actuales de la APS.

El perfil de ese MG debería ser el siguiente:

1. Ejecutar un rol social. Más que un médico que cura enfermedades, el MG debe ser un cuidador de la salud, un educador y consejero de las personas, promoviendo el autocuidado y la conciencia de ellas de los deberes y derechos para salud. Debe ser, por esas características, un agente social valioso, pues por su cercanía a la población, puede entender y manejar mejor las estrategias globales de atención sanitaria. En nuestro país, de acuerdo a los postulados de la reforma, el MG pasa a ser un impulsor de los principios de equidad y solidaridad en salud ${ }^{1,11,12}$. 
2. Desarrollo de competencias. En el mundo occidental se exigen diversas competencias al MG, entre ellas respecto a funciones administrativas, de coordinación entre servicios y de manejo de cierta parte del presupuesto ${ }^{13}$. Entre nosotros, los MG son los encargados de referir los pacientes a los especialistas de acuerdo al AUGE, lo que involucra indirectamente aspectos administrativos y financieros, aunque cumpliendo protocolos bastante rígidos y con costos acotados ${ }^{14}$. Además, deben poseer competencias de gestión y evaluación de la marcha de sus establecimientos y de programación de acciones participativas de la población respecto a medio ambiente, vivienda, salud mental y otras.

3. Liderazgo. En el sistema hospitalario, los estilos de liderazgo son verticales, jerárquicos y autocráticos. Pero en la APS el estilo debe ser participativo, de colaboración interpersonal y con la comunidad, lo que permite una coordinación más fluida con el equipo de salud y con las organizaciones sociales. Así, el MG debe ser el líder de salud en las comunidades 0 pueblos pequeños, coordinando un trabajo en equipo con los demás profesionales y funcionarios de la salud ${ }^{11,15}$.

4. Gestión del modelo bio-psico-social. El MG debe ser el artífice de la promoción de acciones preventivas y de mejoría de los estilos de vida de la comunidad a su cargo. De esa manera puede disminuir o retardar la aparición de las complicaciones de las enfermedades crónicas no transmisibles, y más importante aún, evitar en lo posible la aparición de esas afecciones (prevención primaria). Para ello debe educar, convencer y demostrar a la comunidad que el cambio de sus estilos de vida no saludables los protege frente al riesgo de tales enfermedades. Conjuntamente debe incursionar en las áreas tangenciales que inciden en el riesgo de salud, como alimentación, vivienda, saneamiento ambiental, etc ${ }^{16,17}$.

5. Capacidad técnica y poder de resolutividad. Dada su preparación prioritaria en extensión de los conocimientos médicos, pero no en profundidad, el MG no está en condiciones para resolver definitivamente todos los problemas clínicos en la práctica diaria ${ }^{18}$. Debe por tanto, derivar algunos de ellos a los especialis- tas. En otras ocasiones debe hacerlo por falencias tecnológicas. Se resiente así su poder de resolutividad, lo que ha motivado una crítica frecuente a su desempeño. Sin embargo, las estadísticas que miden la frecuencia de referencias a urgencia y especialidades, han dado resultados variables, que fluctúan entre $15 \%$ a $45 \%$. Estas proporciones son significativas y explican que los consultorios de urgencia y especialidades (CDT) de la red resulten «atochados» frecuentemente.

6. Cultura médica. Se refiere a las características propias de la profesión, como actitud ética, comunicación y lenguaje entendibles y actitudes humanitarias en el quehacer profesional $^{16,19}$. Todas estas propiedades facultan al MG para relacionarse con las personas y la comunidad en los problemas del ambiente, de las enfermedades mentales, drogadicción y otras. Además, puede enfrentar mejor las condiciones de pobreza, contaminación ambiental, enfermedades emergentes en la localidad, y también los problemas administrativos y financieros de su institución.

7. Preparación y entrenamiento en normativas asistenciales existentes. Debe conocer el manejo sanitario institucional, las normas de atención clásicas y la implementación de las garantías explícitas del plan AUGE, entre otras.

8. Acción humanista. Se refiere a mantener un trato amable y de respeto con la persona, sea enferma o sana, por el solo hecho de pertenecer a su comunidad ${ }^{16,19}$

9. Acción sobre la familia. Esta es una obligación del MG, pero que en la práctica no puede cumplir adecuadamente por falencias de formación, tiempo y de facilidades administrativas y técnicas ${ }^{20}$.

10. Gestión y financiación de acciones de la reforma (Auge y otras). Requiere de una capacitación que no se cumple en los planes docentes, lo que determina problemas prácticos diversos.

Este perfil del MG, válido en sus características básicas, no se observa con claridad y propiedad en el accionar de los actuales egresados de las carreras de medicina. Ello ocurre a pesar de los cambios curriculares que hemos mencionado. Por tanto, es importante estudiar y proponer algunas soluciones para reencantar la carrera de MG y 
colocarla de nuevo en su posición de pivote central de la red de salud.

C. Posibles soluciones al problema. Intentaremos analizar algunas de las soluciones que se pueden plantear, algunas de las cuales han sido sugeridas en nuestro medio.

1. Eliminar las deficiencias o restricciones que limitan la incorporación de los MG al sistema público. Las acciones necesarias podrían ser: a) eliminar la inestabilidad laboral, que a la vez es insatisfacción laboral. Para ello se debe establecer una carrera funcionaria, iniciativa que ya está en estudio en el Minsal; b) buscar mecanismos para eliminar la alta rotación de profesionales, pues $40 \%$ de los MG alcanzan una antigüedad menor de un año y $69 \%$ no supera los 3 años en la APS; c) modificar la docencia universitaria relevando la práctica ambulatoria; d) dar importancia a la atención basada en el modelo bio-psico-social; e) incorporar capacitación en psicología, sociología, ciencias de la conducta, etc; f) aumentar los cupos en el sistema público; g) mejorar las condiciones laborales y de remuneración, tornándolas comparables entre las diversas comunas y atendiendo las condiciones de ruralidad y lejanía; h) incorporar incentivos económicos y capacitaciones, cursos, comisiones de estudio y otras acciones de perfeccionamiento profesional.

2. Incorporar a Médicos de Familia. La medicina familiar está ejercida actualmente por un especialista formado mediante un sistema de becas con programas acreditados en las Universidades. El programa dura 2 años y es financiado por el Minsal. Al médico de familia (MF) se le reconocen ventajas en relación al MG, las que serían de tres tipos fundamentales: a) mayor poder de resolución clínica, determinado por una mayor profundidad de conocimientos y una enseñanza especial por 2 años más; b) manejo integral del concepto de Familia. Se trata de médicos preparados para atender las necesidades de las personas y sus familias, realizando una atención integral, sea en los Centros especializados (CESFAM), como en el domicilio. De esa forma se atienden adecuadamente a los miembros de la familia y a las personas que se encuentren postradas; c) orientar a la familia para alcanzar y mantener una vida saludable, evitar enfermedades y resolver o paliar los momentos de crisis en cada etapa de la vida.

Con esta preparación el MF pareciera ser el profesional ideal para la APS, y tal es la opinión de algunos médicos universitarios y de salud pública ${ }^{20}$. Sin embargo, los MF que actualmente están certificados en el país suman poco más de 300, de los cuales sólo 120 ejercían en el sistema público en el año $2004^{21}$. Además, en los últimos 3 años se ha observado un menor interés de los egresados para optar a becas de MF. Las razones de este desinterés parecen ser el trato peyorativo que reciben en los consultorios municipalizados, pues en ellos actúan sólo como MG, desarrollando atención curativa en las 44 horas contratadas.

3. Incorporar especialistas básicos a la APS. Algunos autores han propuesto destinar una parte de la jornada de los becarios de especialidades básicas (Medicina Interna, Pediatría, Ginecología, Psiquiatría) a la APS. Para ello, realizarían la mitad de su jornada de $44 \mathrm{~h}$. en su labor de aprendizaje de la especialidad, y el resto, en atención ambulatoria en los consultorios municipalizados. Esto les permitiría familiarizarse con los aspectos preventivos y de rehabilitación de la salud. Sin embargo, esta proposición ha sido rechazada por la ASOFAMECH y sus Escuelas de Graduados, aduciendo que con una jornada parcial no se puede completar a cabalidad la formación teórica y práctica de la beca de especialidad.

Otro mecanismo propuesto sería la incorporación decidida de especialistas básicos, una vez certificados, en la APS de sus respectivos servicios regionales. Reforzarían la atención ambulatoria en base a equipos de 3 ó 4 especialistas de diferentes disciplinas en algunos consultorios de comunas con gran número de habitantes. Así podrían resolver problemas y referencias de los MG de las comunas aledañas con menos recursos. Realizarían de esta manera un mecanismo de filtro de interconsultas a los consultorios de especialidades o subespecialidades adosados a los hospitales base, que actualmente permanecen atochados con esas solicitaciones. Este sistema está en 
funciones en el Reino Unido y exhibe buenos resultados ${ }^{22}$.

\section{DisCUSIÓN}

Hemos realizado un análisis de las posibles causales de la desproporción creciente entre MG y especialistas, en favor de éstos últimos. El desarrollo científico y tecnológico ha estimulado la especialización, incentivada también por la medicalización de la sociedad y el progreso económico-social. Se premia la capacidad resolutiva parcelar en desmedro de la indispensable visión de conjunto del estado de salud del individuo. El modelo que tienden a reproducir las recientes generaciones de médicos es el de especialista 0 ultraespecialista, que trabaja con múltiples tecnologías y obtiene grandes éxitos.

Sin embargo, la motivación ética y el deseo de servir de los estudiantes de medicina se mantienen intactos. Más de $60 \%$ de los egresados estarían dispuestos a servir como MG, pero ello no ocurre en la práctica por las razones ya analizadas, puesto que cada año son más los egresados que postulan a los cupos de MG del Minsal sin alcanzarlos por ser reducidos ${ }^{23}$.

Tal vez el perfil del MG necesario para la Reforma no es el adecuado en la formación universitaria, a pesar de los excelentes cambios curriculares adoptados en los últimos años. Tal vez una fuerte capacitación por 3 a 6 meses a los egresados que se contraten como $\mathrm{MG}$ en el sistema público (y también, por qué no, en el privado) permitiría obtener el perfil deseado ${ }^{24}$.

Otra posible solución sería contar con internados electivos para MG y preespecialistas básicos,

\section{REFERENCIAS}

1. Montero J, Muñoz F, Rosselot E, Valdivieso V, BARBA R. La Medicina general en la organización médica actual. Rev Méd Chile 1996; 124: 1006-14.

2. Román O, AcuÑa M, Señoret M. Disponibilidad de médicos en Chile al 2004. Rev Méd Chile 2006, 134: 1057-64.

3. Diario Oficial Ley 19.937. Modifica el Dto. Ley 2762 de 1979 con la finalidad de establecer una nueva concepción de la Autoridad Sanitaria, pero ello atentaría contra la preparación integral del excelente egresado con el que contamos ${ }^{8,9}$.

De este análisis surge también la ausencia de relación que existe entre el número de egresados de nuestras universidades y los cupos en las plantas del Sistema Público Ministerial y de las Municipalidades. No existen suficientes vacantes para los egresados que manifiestan interés por el generalato médico. Tampoco parecen suficientes las horas de planta para algunas especialidades y subespecialidades $^{21}$. Por ello, cobra gran importancia reconstituir una relación docente-asistencial fuerte, armónica y con una finalidad concreta de servicio público. Ello permitiría encauzar mejor la producción nacional universitaria y adecuarla a las necesidades reales de la población, tanto en el sector público como en el privado. De otra manera vamos a pasos agigantados a determinar una sobresaturación de médicos en el país, especialmente de especialistas y abrir las puertas al fantasma de la cesantía médica ${ }^{2}$. Es preciso construir un perfil de MG que se adapte ahora a las necesidades y modelos de atención de salud del país, de acuerdo a los principios de la Reforma. De otra manera, la imagen del MG con exceso de trabajo rutinario y ausencia de formación profesional adecuada, aparece «quijotesca» e incluso, incompatible con las posibilidades de una vida digna ${ }^{25}$

Creemos que es fundamental seguir formando un profesional no sólo técnica y científicamente preparado, sino también orientado a las necesidades del bien común con una actitud humanista y con principios acordes con las declaraciones ético-morales impresas en los códigos, pero también, como apunta el Dr. Parada ${ }^{26}$, en la mente del profesional con letras de fuego.

distintas modalidades de gestión y fortalece la participación ciudadana. Febrero, 2004.

4. Diario Oficial Ley 19378. Estatuto de atención primaria de salud municipal. Abril 1995.

5. Diario Oficial. Ley 19.664. Establece normas especiales para profesionales funcionarios que indica de los servicios de salud y modifica la Ley 15.076. Febrero 2000.

6. Carabantes J. Sistema público de salud y tipo de médicos que éste necesita. Rev Méd Chile 1997; 125: $24-36$ 
7. JADRESIC A. Hacia una política de desarrollo de recursos humanos para la salud. Rev Méd Chile 1991; 119: 589-99.

8. RosSeLot E. Reorganización de la educación médica. Aspectos esenciales de la reestructuración de la Facultad de Medicina de la Universidad de Chile. Rev Méd Chile 1997; 125: 783-91.

9. Rosso P, Velasco N, Moreno R. Reforma del curriculum de pregrado en la Escuela de Medicina de la Pontificia Universidad Católica de Chile: objetivos, metodología y estado de avance. Rev Méd Chile 1997; 125: 796-807.

10. Román O. Antecedentes sobre formación universitaria. En «a crisis de la Profesión Médica». Santiago: Ed. Abarca. 2001; 93-98.

11. Román O, Solar G. Proposición global de reforma del Sistema de Salud de Chile. Cuad Med Soc 1993; 34: 23-37.

12. IpINZA M. Algunas reflexiones acerca de la situación actual de la atención primaria y del nivel primario de atención. Colegio Médico. Serie Publicaciones Técnicas № 9, Marzo 1997.

13. Sistema de Salud del Reino Unido. Personal requerido para el sistema de salud inglés. Informe 1999.

14. Diario Oficial. Ley 19.996. Del régimen de garantías en salud. Septiembre, 2004.

15. Cohen W. El arte de ser líder. Buenos Aires. Ed. Atlántida. 1990

16. The Hastings Center. The Goals of Medicine: Setting new priorities The Hastings Center Report. Nov-Dec, 1996.

17. Paganini JM. La salud en las grandes ciudades y los sistemas locales de salud. Bol Of Sanit Panam 1989; 107: 65-73.
18. Academia Chilena de Medicina. Control de calidad en Medicina. Posición de la Academia Chilena de Medicina. Rev Méd Chile 2001; 129: 819-21.

19. BASCUÑán L Cambios en la relación médicopaciente y nivel de satisfacción de los médicos. Rev Méd Chile 2005; 133: 11-6.

20. Sociedad Científica de Medicina Familar y General DE CHILE. La medicina familiar en la reforma de salud chilena. Rev Ch Med Familiar 2000; 1: 2532.

21. AcUÑa M. Estado de formación de los médicos cirujanos y especialistas en los Servicios de Salud. Informe de resultados al año 2004. Monografía. Ministerio de salud. Febrero, 2005.

22. Department of Health British Crown. Driving efficiency in the National Health Service. 1977 December, pags 1-4.

23. García Huidobro D, Núñez F, Vargas P, AstudiLo S, HITSChFELD M ET AL. Expectativas de estudiantes de medicina de pregrado en relación al perfil del médico esperado. Rev Méd Chile 2006; 134: 94754.

24. Reyes H. ¿Podemos mejorar la atención médica primaria del adulto? Rev Méd Chile 1992; 120: 571-6.

25. Velasco C. El diagnóstico y proyección de la profesión de médico. En: Perfil del profesional de la salud en el siglo XXI. Edición Universidad de Chile. 1995; págs 103-10.

26. Parada M. Estudios de Post Título y de Post Grado en Medicina. En Perfil del profesional de la salud en el siglo XXI. Edición Universidad de Chile 1995; págs. 111-17. 